Fúlvio Borges Nedel',II

Luiz Augusto Facchini'

Miguel Martín-Mateo"

Lúcia Azambuja Saraiva

Vieira"IIIIV

Elaine Thumé'

\section{Programa Saúde da Família e condições sensíveis à atenção primária, Bagé (RS)}

\section{Family Health Program and ambulatory care-sensitive conditions in Southern Brazil}

\section{RESUMO}

OBJETIVO: Condições sensíveis à atenção primária (CSAP) são problemas de saúde atendidos por ações do primeiro nível de atenção. A necessidade de hospitalização por essas causas deve ser evitada por uma atenção primária oportuna e efetiva. O objetivo do estudo foi estimar a probabilidade do diagnóstico de CSAP em pacientes hospitalizados pelo Sistema Único de Saúde.

MÉTODOS: Estudo transversal com 1.200 pacientes internados entre setembro/2006 e janeiro/2007 em Bagé (RS). Os pacientes responderam a questionário aplicado por entrevistadoras, sendo classificados segundo o modelo de atenção utilizado previamente à hospitalização. As CSAP foram definidas em oficina promovida pelo Ministério da Saúde. Analisaram-se variáveis demográficas, socioeconômicas, de situação de saúde e relativas aos serviços de saúde utilizados. A análise multivariável foi realizada por modelo de Poisson, seguindo modelo teórico hierárquico de determinação da hospitalização segundo sexo e modelo de atenção.

Epidemiologia. Departamento de Medicina Social. Faculdade de Medicina da Universidade Federal de Pelotas. Pelotas, RS, Brasil

" Grups de Recerca d'Amèrica i Àfrica Llatines. Unitat de Bioestadística. Facultat de Medicina. Universitat Autònoma de Barcelona. Barcelona, Espanha

III Centro de Ciências da Saúde. Universidade da Região da Campanha. Bagé, RS, Brasil

iv $7^{\text {a }}$ Coordenadoria Regional de Saúde. Secretaria da Saúde do Estado do Rio Grande do Sul. Bagé, RS, Brasil

Correspondência | Correspondence:

Fúlvio Borges Nedel

Departamento de Medicina Social

Faculdade de Medicina

Universidade Federal de Pelotas

R. Marechal Deodoro, 1160 - $3^{\circ}$ piso - Centro

Caixa Postal 464

96020-220 Pelotas, RS, Brasil

E-mail: fulvionedel@yahoo.com.br
RESULTADOS: O total de 42,6\% das internações foi por condições sensíveis à atenção primária. A probabilidade de que o diagnóstico principal de internação seja por uma dessas condições aumenta com as características: ser do sexo feminino, ter idade menor de cinco anos, ter escolaridade menor de cinco anos, ter sido hospitalizado no ano anterior à entrevista, ter consulta médica na emergência, estar internado no hospital universitário. Associaram-se à probabilidade de CSAP: (a) mulheres: faixa etária, escolaridade, tempo de funcionamento da unidade de saúde, residir em área de saúde da família, ser usuária do Programa Saúde da Família, consulta médica na emergência no mês anterior à pesquisa e hospital de internação; (b) homens: faixa etária, ter sofrido outra internação no ano anterior à entrevista e o hospital de internação.

CONCLUSÕES: As condições sensíveis à atenção primária permitem identificar grupos carentes de atenção à saúde adequada. Embora o estudo não permita inferências sobre o risco de internação, as análises por sexo e modelo de atenção sugerem que o Programa Saúde da Família é mais eqüitativo que a atenção básica tradicional.

DESCRITORES: Programa Saúde da Família. Atenção Primária à Saúde. Serviços de Saúde, utilização. Hospitalização. Fatores Socioeconômicos. Estudos Transversais. 


\begin{abstract}
OBJECTIVE: Ambulatory care-sensitive conditions (ACSC) are health problems managed by actions at the first level of care. The need for hospitalization by these causes is avoidable through an effective and proper primary health care. The objective of the study was to estimate ACSC among patients hospitalized by the Sistema Único de Saúde (Brazilian Health System).
\end{abstract}

METHODS: Hospital-based cross-sectional study involving 1,200 inhabitants of Bagé (Southern Brazil) who were inpatients between September/2006 and January/2007. The patients answered a questionnaire applied by interviewers and were classified according to the model of attention utilized prior to hospitalization. ACSC were defined in a workshop promoted by the Ministry of Health. The variables analyzed included demographic and socioeconomic characteristics, health and health services utilized. Multivariate analysis was conducted by the Poisson model, according to a hierarchical conceptual framework, stratified by sex and model of care.

RESULTS: ACSC accounted for $42.6 \%$ of the hospitalizations. The probability that the main diagnosis for hospitalization is considered an ACSC is greater among women, children under five years of age, individuals with less then five years of schooling, hospitalization in the year prior to the interview, emergency room consultation, and being an inpatient at the university hospital. Among women, ACSC are associated with age, educational level, length of time the health center has been in existence, living in an area covered by the Programa Saúde da Família (Family Health Program), use of this service, emergency room consultation during the month prior to the interview and hospital to which patient was admitted. For men, it was associated with age, have undergone another hospitalization in the year prior to the interview and hospital to which patient was admitted.

CONCLUSIONS: Analysis of ACSC allows identifying groups with inadequate access to primary health care. Although we could not infer an effect on the risk of hospital admission, analysis by sex and model of care suggests that Family Health Program is more equitable than "traditional" primary health care.

DESCRIPTORS: Family Health Program. Primary Health Care. Health Services, utilization. Hospitalization. Socioeconomic Factors. CrossSectional Studies.

\title{
INTRODUÇÃO
}

A atenção primária à saúde é a principal proposta de modelo assistencial da Organização Mundial da Saúde, visando a melhoria dos indicadores de saúde, redução das brechas de morbi-mortalidade, e um consumo mais racional da tecnologia biomédica, para maior eficiência ao gasto no setor. ${ }^{a}$ No Brasil, o Sistema Único de Saúde (SUS) e o Programa Saúde da Família (PSF) seguem os mesmos princípios, buscando a reorientação do modelo assistencial a partir da atenção primária.

A avaliação e o monitoramento de ações e resultados são fundamentais na adequação das políticas e ações implementadas. Entre os esforços governamentais realizados nesse sentido, citam-se o Sistema de Informação da Atenção Básica, o Programa de Expansão e Consolidação da Saúde da Família, ${ }^{13}$ o Pacto dos Indicadores da Atenção Básica ${ }^{19} \mathrm{e}$ editais de financiamento à pesquisa. Entretanto, faltam instrumentos e estudos de avaliação do impacto da atenção primária sobre a saúde da população. ${ }^{15} \mathrm{O}$ Sistema de Informação da Atenção Básica é útil para a organização das equipes de saúde da família, ${ }^{1}$ mas oferece limitações à pesquisa avaliativa, além de não ser usado para registrar as informações sobre a atenção básica "tradicional” (não-PSF), impedindo a comparação entre os modelos de atenção.

a Starfield B. Atenção primária: equilíbrio entre necessidades de saúde, serviços e tecnologia. Brasília: Unesco/Ministério da Saúde; 2002. 
A avaliação do Programa de Expansão e Consolidação da Saúde da Família restringe-se aos municípios com mais de cem mil habitantes. Ao "Pacto dos Indicadores" falta uma medida agregada que permita uma avaliação mais integral da atenção básica.

De outra parte, as internações por condições sensíveis à atenção primária (CSAP - Ambulatory Care Sensitive Conditions) têm se expandido como indicador indireto do acesso à atenção oportuna e efetiva no primeiro nível de atenção à saúde. A idéia é que a resolubilidade da atenção primária à saúde deve se refletir na diminuição das internações hospitalares por um grupo de causas específicas. ${ }^{7,12,14, a, b}$ CSAP são problemas de saúde atendidos por ações características do primeiro nível de atenção. As hospitalizações por CSAP são um indicador indireto da efetividade do sistema de saúde, nessa instância de atenção, pressupondo que as pessoas internadas por CSAP não receberam atenção de saúde efetiva em momento oportuno, levando a um agravamento de sua condição clínica, exigindo a hospitalização. ${ }^{9,10}$

O indicador foi criado ao final da década de 1980 nos Estados Unidos para avaliar o impacto da falta de acesso a serviços de atenção primária. ${ }^{5,6,20}$ Posteriormente, em países com acesso universal, como a Espanha, passou a ser usado para comparar a efetividade de modelos de atenção primária à saúde. ${ }^{4,8}$ No Brasil, realizaram-se estudos no Ceará e Minas Gerais. ${ }^{\mathrm{a}}$

Por princípio de eqüidade, a implantação do PSF inicia em áreas de maior pobreza, cujas populações também sofrem maior ocorrência de doenças e limitações de acesso a serviços, entre eles os de saúde. Assim, as características socioeconômicas da população são necessárias à interpretação do efeito do modelo de atenção sobre as hospitalizações por CSAP, especialmente quando a cobertura do PSF não é universal. $\mathrm{Na}$ falta de tais variáveis, a agregação das condições dos indivíduos pode servir de proxy.

As CSAP permitem identificar, de modo objetivo e comparável, parcelas da população carentes de atenção primária à saúde adequada, pelo que se presumem convenientes ao SUS. Assim, este estudo procura responder à seguinte pergunta: comparado a outros modelos de organização da atenção, o PSF se associa a uma menor proporção de CSAP entre a população hospitalizada? O objetivo do presente estudo foi estimar a probabilidade do diagnóstico de CSAP entre os residentes hospitalizados pelo SUS, segundo o modelo de atenção utilizado nas consultas prévias à internação.

\section{MÉTODOS}

A população do município de Bagé estimada para 2006 era de 122.461 habitantes, $82 \%$ vivendo na zona urbana. Conta com três hospitais (um hospital do exército, não conveniado ao SUS), 470 leitos hospitalares conveniados ao SUS (um leito para cada 3,8 mil habitantes) e 21 unidades básicas de saúde (UBS), 13 delas do PSF (52\% da população).

Todas as hospitalizações de residentes do município que internaram pelo SUS nos hospitais do município entre 16 de setembro de 2006 e 15 de janeiro de 2007 foram analisadas. Os pacientes (ou seus responsáveis, no caso das crianças) foram entrevistados durante a internação hospitalar, por entrevistadoras treinadas, durante todos os dias da semana.

O questionário abordou informações demográficas, socioeconômicas e relacionadas ao uso de serviços de saúde. Os diagnósticos de internação e alta foram coletados nos registros hospitalares, após a alta do paciente.

Foram excluídas as internações obstétricas, as internações que terminaram em óbito, os pacientes transferidos da unidade de terapia intensiva (UTI) a outros hospitais e as pessoas sem condições de responder o questionário, segundo avaliação da entrevistadora, além daquelas que se recusaram a participar. Pacientes internados na UTI eram entrevistados após a transferência para o quarto. Não houve limite de idade para inclusão no estudo.

O número mínimo de hospitalizações necessárias para detectar uma probabilidade de CSAP entre os pacientes residentes em área do PSF diferente em 10\% de outras áreas do município foi de 868 pessoas, segundo a aproximação arcoseno, ${ }^{17}$ com parâmetros alfa $=5 \%$, beta $=20 \%$, perdas $=10 \%$, proporção global de CSAP estimada em 46\%, relação PSF: não-PSF de 1:1. Acrescentando-se $30 \%$ para conferir maior estabilidade estatística ao ajuste para fatores de confusão, o cálculo amostral resultou em no mínimo 1.129 sujeitos.

A digitação e parte do processamento dos dados foram realizados no programa EpiData Entry.

A avaliação das UBS foi efetuada em grupo de discussão com o Secretário de Saúde e a equipe de coordenação da atenção básica do município, sobre os critérios: compromisso com o usuário, trabalho em equipe, intersetorialidade, integralidade, eqüidade, trabalho comunitário e organização da atenção. Ao final da discussão, cada UBS recebeu uma nota de zero a dez sobre cada um dos critérios.

\footnotetext{
a Mendes EV. A atenção primária à saúde no SUS. Fortaleza: Escola de Saúde Pública do Ceará; 2002.

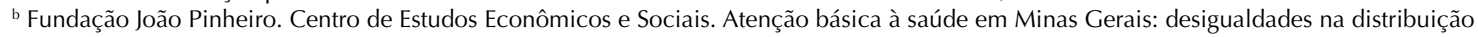
de recursos financeiros e na prestação de serviços básicos após a introdução do piso da atenção básica (PAB) [internet]. Belo Horizonte [citado 2004 nov 21]. Disponível em: http://www.fjp.gov.br/produtos/cees/ Atencao_Basica_em_Minas_Gerais.pdf ${ }^{c}$ Ministério da Saúde. DATASUS. Informações de saúde [internet]. Brasília; 2007 [citado 2007 fev 20]. Disponível em: http://tabnet.datasus.gov.br
} 
O desfecho em estudo é a proporção de CSAP entre as hospitalizações analisadas. A lista de códigos da $10^{\mathrm{a}}$ revisão da Classificação Internacional de Doenças (CID-10) considerados CSAP foi definida em oficina de trabalho promovida pelo Ministério da Saúde, em dezembro de $2005^{\text {a }}$ (Tabela 1). Nesse processo, inspirado no trabalho de Caminal et al, ${ }^{11}$ um grupo de especialistas respondeu a questões sobre a freqüência, transcendência, sensibilidade a ações da atenção primária à saúde, facilidade diagnóstica, necessidade de internação no caso de desenvolvimento da doença e a influência de incentivos financeiros sobre os códigos registrados, de uma lista de causas pré-selecionadas. A variável de desfecho foi criada no programa EpiData Analysis, considerando o CID registrado no diagnóstico de alta. Definiu-se como usuário do PSF o morador de Área de Saúde da Família e que consultou no último mês nessa unidade (pelo motivo da internação ou não).

Posteriormente à descrição e análise bivariada dos dados, realizou-se una análise multivariável por modelo de Poisson, seguindo um esquema teórico hierárquico de determinação da hospitalização por CSAP (Tabela 2). Nesse modelo encontram-se, em nível mais distal, determinantes da condição de saúde e do uso de serviços; em nível intermediário, descritores da condição de saúde e uso de serviços pré-hospitalares e, em nível proximal ao desfecho, descritores dos serviços hospitalares utilizados, bem como do desempenho dos serviços de atenção básica do município.

Todas as variáveis do nível de determinação analisado, iniciando pelo nível mais distal ao desfecho, foram colocadas na equação e aquelas com $\mathrm{p} \geq 0,2$ eliminadas, passo-a-passo. Cada nível subseqüente incorpora as variáveis mantidas anteriormente; as medidas de efeito e valor-p relatados para cada variável são as encontradas no seu nível de análise. A análise multivariável foi realizada para todo o conjunto de indivíduos e para cada estrato de sexo e de modelo de atenção. Salvo indicação, os valores-p apresentados foram obtidos pelo teste exato de Fisher ou, na comparação de médias, pelo teste $t$ de Student ou ANOVA; a análise foi realizada no programa Stata 9.0.

Os participantes assinaram um Termo de Consentimento Livre e Esclarecido antes de responder ao questionário. O estudo foi aprovado pelo Comitê de Ética em Pesquisa da UFPel.

\section{RESULTADOS}

Entre 16/9/2006 e 15/1/2007 foram registradas nos hospitais de Bagé 1.446 internações não-obstétricas de residentes no município, a serem pagas pelo SUS.
A proporção de recusas foi de 6,6\% (96 casos), grupo com idade média 19,8 anos maior que os entrevistados $(\mathrm{p}<0,001)$ e sem diferença significativa de sexo. Em 47 casos $(3,5 \%)$ o entrevistado informou residir em outro município, sendo excluído do estudo; esses casos não diferiram dos demais por hospital, idade ou sexo. Dos 1303 sujeitos que concordaram em participar do estudo, 103 (7,9\%) internaram mais de uma vez, restando para análise 1.200 casos únicos de internação hospitalar. As variáveis com maior proporção de dados ausentes foram: diagnóstico de alta $(6,0 \%)$, renda familiar (7,4\%), outra internação no ano anterior $(8,4 \%)$ e recomendação de internar na consulta por plano de saúde $(5,4 \%)$.

Os entrevistados tinham em média 5,7 $(\mathrm{dp}=4,0)$ anos de estudo e viviam em zona urbana $(96,2 \%)$, com renda familiar média (no último mês) de $\mathrm{R} \$ 730,46$ $(\mathrm{dp}=564,60)$ e renda familiar per capita média de $\mathrm{R} \$$ $226,65(\mathrm{dp}=198,59)$, pertencendo às categorias $\mathrm{C}$ e D do Critério de Classificação Econômica da Associação Brasileira de Empresas de Pesquisas $(83,4 \%)$. Os quartis de idade foram: 4, 35,5 e 58 anos. A proporção de sexo e faixa etária foi diferente entre os hospitais: no hospital filantrópico, a maioria dos pacientes foi do sexo masculino $(54,4 \%)$ e a distribuição por faixa etária mais homogênea, enquanto no hospital universitário internam mais mulheres $(56,9 \%)$ e adultos ou idosos, com idade média 9,7 anos maior (valores de $\mathrm{p}<0,001)$. Os hospitais diferiram nas especialidades dos médicos responsáveis pelas internações (segundo a informação do paciente): no hospital universitário foi menor a proporção de internações por pediatras (26,0\%, contra $33,3 \%$ no filantrópico) e cirurgiõesgerais $(1,2 \%$, contra $13,6 \%)$ e maior a de internações realizadas por outros especialistas $(39,5 \%$ contra $20,3 \%$ ); clínicos gerais internaram $29 \%$ dos casos e ginecologistas 4\%, em ambos hospitais.

Segundo os diagnósticos de alta, foram registradas no período de estudo 480 internações por CSAP (42,6\%; IC95\% [39,7;45,4]), semelhante aos 43,0\% encontrados ao usar o diagnóstico do momento da internação. A renda familiar média foi R \$ 69,82 menor entre as pessoas internadas por CSAP que entre as internadas pelas demais causas $(p=0,007)$; a escolaridade média foi semelhante entre os grupos (diferença de 0,3 anos; $p=0,2$ ).

A Tabela 3 apresenta a relação entre modelo de atenção e CSAP como diagnóstico de internação. Não houve diferenças significativas segundo o modelo de atenção ou uso da unidade de saúde de referência. Entretanto, independentemente da população de análise, as proporções de CSAP foram maiores no PSF ou quando o paciente consultou um médico na sua UBS no último mês.

a Ministério da Saúde. Departamento de Atenção Básica. Oficina de trabalho para elaboração de uma lista brasileira de hospitalização por condições sensíveis à atenção primária. Belo Horizonte, MG, 9/12/2005. (dados inéditos) 
Tabela 1. Lista brasileira de condições sensíveis à atenção básica.

\begin{tabular}{|c|c|}
\hline Grupo de causa & Código \\
\hline 1. Doenças preveníveis por imunização e condições evitáveis & $\begin{array}{l}\text { A33 - A35, B05, G00.0, A37, A36, A50, A51 A53, } \\
\text { A15.0-A15.3, A16.0-A16.2, A17.0, A15.4 - A15.9, A16.3 } \\
\text { - A16.9, A17.1-A17.9, A18, A19, A95, B50-B54, B16 }\end{array}$ \\
\hline 2. Gastroenterites Infecciosas e complicações & A00-A09, E86 \\
\hline 3. Anemia por deficiência de ferro & D50 \\
\hline 4. Deficiências Nutricionais & E40-E46, E50-E64 \\
\hline 5. Infecções de ouvido, nariz e garganta & H66, J00-J03, J06, J31, I00-I02 \\
\hline 6. Pneumonias bacterianas & $\mathrm{J} 13, \mathrm{~J} 14, \mathrm{~J} 15.2-\mathrm{J} 15.4, \mathrm{~J} 15.8, \mathrm{~J} 15.9, \mathrm{~J} 17, \mathrm{~J} 18$ \\
\hline 7. Asma & $\mathrm{J} 45, \mathrm{~J} 46$ \\
\hline 8. Doença pulmonar obstrutiva crônica & $\mathrm{J} 20, \mathrm{~J} 21, \mathrm{~J} 40-\mathrm{J} 44, \mathrm{~J} 47$ \\
\hline 9. Hipertensão & $110, \mid 11$ \\
\hline 10. Angina pectoris & 120,124 \\
\hline 11. Insuficiência cardíaca & I50, J81 \\
\hline 12. Doenças cerebrovasculares & I60-I69 \\
\hline 13. Diabetes melitus & E10-E14 \\
\hline 14. Epilepsias & G40, G41 \\
\hline 15. Infecção no rim e trato urinário & N00, N10-N12, N15.9, N39.0, N30, N34 \\
\hline 16. Infecção da pele e tecido subcutâneo & A46, L01-L04, L08 \\
\hline 17. Doença Inflamatória órgãos pélvicos femininos & N70-N73, N75, N76 \\
\hline 18. Úlcera gastrointestinal com hemorragia e/ou perfuração & $\begin{array}{l}\text { К25.0-К25.2, К25.4-К25.6, К26.0-К26.2, К26.4-К26.6, } \\
\text { К27.0-К27.2, К27.4-К27.6, К28.0-К28.2, К28.4-К28.6 }\end{array}$ \\
\hline 19. Neoplasia maligna do útero & C53, C55 \\
\hline 20. Doenças relacionadas ao pré-natal e parto & O23, P00, P35.0, P70.0, P70.1, B20-B24 \\
\hline
\end{tabular}

Fonte: Ministério da Saúde. Departamento de Atenção Básica. Oficina de trabalho para elaboração de uma lista brasileira de hospitalização por condições sensíveis à atenção primária. Belo Horizonte, MG, 9/12/2005.

A análise bivariada mostrou maior probabilidade de que o diagnóstico principal de internação fosse uma CSAP em: mulheres, crianças menores de cinco anos de idade, pessoas com menor grau de escolaridade, mais pobres, que sofreram outra hospitalização no ano anterior e nas que consultaram no mês anterior por outro motivo além do que levou à internação. Também se associaram o hospital de internação e a especialidade do médico.
A internação foi recomendada em 42,8\% dos casos no $\mathrm{PSF}$, contra $61,3 \%$ nos não-PSF ( $\mathrm{RP}=0,70$; IC 95\% $[0,57 ; 0,86])$.

Embora a recomendação de internar na consulta por plano de saúde tenha alcançado $\mathrm{p}=0,2$, o número de observações foi pequeno $(n=37)$ e a variável não foi incluída no modelo multivariado.

Tabela 2. Modelo hierárquico de análise das variáveis no estudo.

\begin{tabular}{|c|c|}
\hline Nível de determinação & Variável \\
\hline \multirow[t]{2}{*}{1} & Demográfica, socioeconômica \\
\hline & $\begin{array}{l}\text { Sexo, faixa etária, escolaridade, renda familiar per capita, posse de bens de consumo (CCEB-ABEP), } \\
\text { zona de residência (rural ou urbana), tempo de residência no domicílio atual. }\end{array}$ \\
\hline \multirow[t]{2}{*}{2} & Condição de saúde, uso de serviços \\
\hline & $\begin{array}{l}\text { Outra internação nos últimos } 12 \text { meses; outra consulta nos últimos } 30 \text { dias; tempo de funcionamento } \\
\text { do PSF; ser usuário do PSF; modelo de atenção nas consultas nos últimos } 30 \text { dias pelo problema } \\
\text { que gerou a internação; recomendação de internação, nessas consultas. }\end{array}$ \\
\hline \multirow[t]{2}{*}{3} & Uso de serviços, desempenho dos serviços \\
\hline & $\begin{array}{l}\text { Hospital de internação; especialidade do médico responsável pela internação; avaliação dos } \\
\text { serviços pelo gestor. }\end{array}$ \\
\hline \multicolumn{2}{|r|}{ Desfecho: hospitalização por condições sensíveis à atenção primária } \\
\hline
\end{tabular}

CCEB-ABEP: Critério de Classificação Econômica Brasil, da Associação Brasileira de Empresas de Pesquisa 
Tabela 3. Prevalência hospitalar de condições sensíveis à atenção primária e uso de serviços de saúde. Bagé, RS, $2006-2007$.

\begin{tabular}{|c|c|c|c|}
\hline População / Variável & $\mathrm{N}(\%)$ & $\begin{array}{c}\text { Prevalência } \\
\text { CSAP }\end{array}$ & RP (IC 95\%)* \\
\hline Todos indivíduos & 1.200 & & \\
\hline Modelo de atenção do serviço de referência & \multicolumn{3}{|c|}{ Missing $6,0 \%$} \\
\hline PSF & $624(52,0)$ & 43,1 & 1 \\
\hline Atenção básica tradicional & $386(32,2)$ & 42,4 & $0,98(0,84 ; 1,15)$ \\
\hline Outro & $190(15,8)$ & 41,1 & $0,95(0,78 ; 1,16)$ \\
\hline Consultou exclusivamente na unidade de saúde de referência & \multicolumn{3}{|c|}{ Missing $6,0 \%$} \\
\hline Não & $1084(90,3)$ & 41,8 & 1 \\
\hline Sim & $116(9,7)$ & 49,1 & $1,17(0,96 ; 1,44)$ \\
\hline Que consultaram na sua unidade de saúde de referência & 324 & & \\
\hline Modelo de atenção da unidade de saúde de referência & \multicolumn{3}{|c|}{ Missing $7,1 \%$} \\
\hline PSF & $194(59,9)$ & 48,3 & 1 \\
\hline Outro & $130(40,1)$ & 45,5 & $0,94(0,74 ; 1,20)$ \\
\hline Consultaram somente na sua unidade de saúde de referência & 110 & & \\
\hline Modelo de atenção da unidade de saúde de referência & \multicolumn{3}{|c|}{ Missing 5,4\% } \\
\hline PSF & $48(43,6)$ & 54,6 & 1 \\
\hline Outro & $62(56,4)$ & 46,7 & $0,86(0,58 ; 1,25)$ \\
\hline Residentes em Área de Saúde da Família & 623 & & \\
\hline Usuário do PSF (1 missing) & \multicolumn{3}{|c|}{ Missing 5,9\% } \\
\hline Não & $400(64,2)$ & 40,9 & 1 \\
\hline Sim & $223(35,8)$ & 47,3 & $1,16(0,96 ; 1,40)$ \\
\hline \multicolumn{4}{|l|}{ Exclui pacientes sem informação sobre unidade de saúde de referência } \\
\hline Consulta na unidade de saúde de referência & 953 & Missing $6,6 \%$ & \\
\hline Não & $652(68,4)$ & 40,8 & 1 \\
\hline Sim & $301(31,6)$ & 47,2 & $1,15(0,99 ; 1,34)$ \\
\hline Consultou somente na unidade de saúde de referência & 953 & Missing 6,6\% & \\
\hline Não & $849(89,1)$ & 41,9 & 1 \\
\hline Sim & $104(10,9)$ & 50,0 & $1,19(0,97 ; 1,47)$ \\
\hline
\end{tabular}

* Modelo de Poisson com variância robusta

PSF: Programa Saúde da Família

CSAP: Condições sensíveis à atenção primária

A Tabela 4 apresenta a descrição e análise bivariada das variáveis que entraram no modelo de ajuste, bem como a análise multivariável, em todos os sujeitos de estudo e por estratos de sexo. Observa-se que ser usuário do PSF, o tempo de funcionamento da UBS e, principalmente, o tempo como PSF, estiveram próximos do limite de significância estatística. Entre os critérios de desempenho avaliados pelo gestor e coordenadores da atenção básica, o que esteve mais próximo da associação com uma CSAP como causa da internação foi a "organização da atenção" $(\mathrm{p}=0,1)$.

Observa-se na Tabela 4 que a proporção de CSAP entre as mulheres foi $28 \%$ maior que entre os homens. A faixa etária com maior risco de se encontrar uma CSAP entre os pacientes hospitalizados pelo SUS foi a dos menores de cinco anos, diminuindo a partir de então e voltando a aumentar entre os pacientes com 50 ou mais anos de idade. A proporção de CSAP diminuiu com o aumento da escolaridade dos pacientes $(\mathrm{p}<0,001)$. A mesma Tabela mostra que quando o serviço de referência é do PSF, a probabilidade de diagnóstico de CSAP foi $15 \%$ menor $(p=0,08)$. O hospital foi o principal fator associado. Os pacientes cuja UBS de referência recebeu nota acima da mediana no critério "trabalho em equipe" apresentaram probabilidade $19 \%$ menor de que a causa de internação fosse uma $\operatorname{CSAP}(p=0,06)$. A probabilidade de diagnóstico de CSAP aumentou para: sexo feminino, idade menor de cinco anos, escolaridade menor de cinco anos, hospitalização no ano anterior, consulta na emergência, internar no hospital universitário.

Ainda na Tabela 4, as principais diferenças entre sexos referiram-se ao modelo de atenção. A proporção de CSAP foi 33\% menor entre as mulheres residentes em área do PSF, mas $32 \%$ maior entre as que consultaram no 


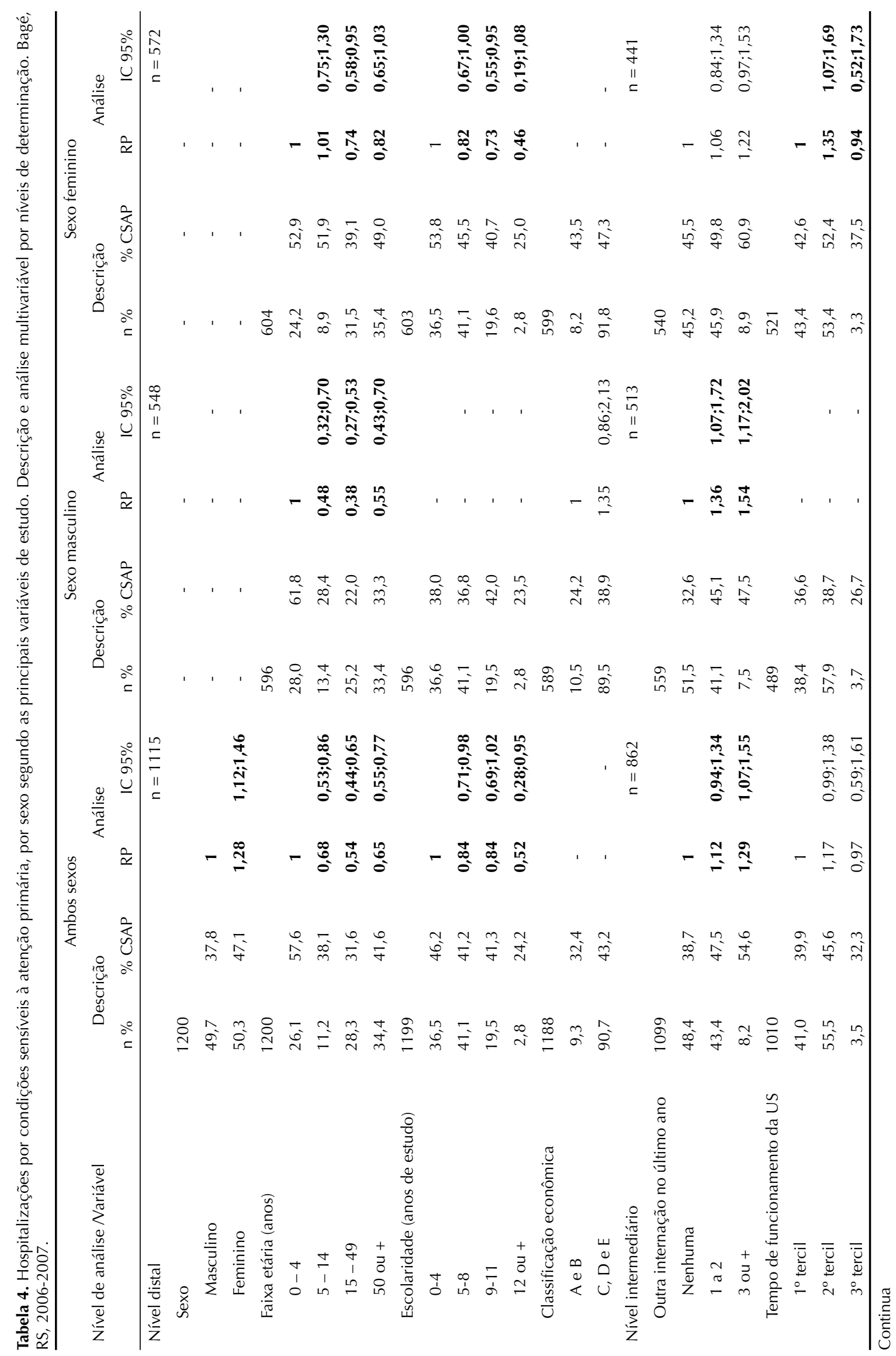




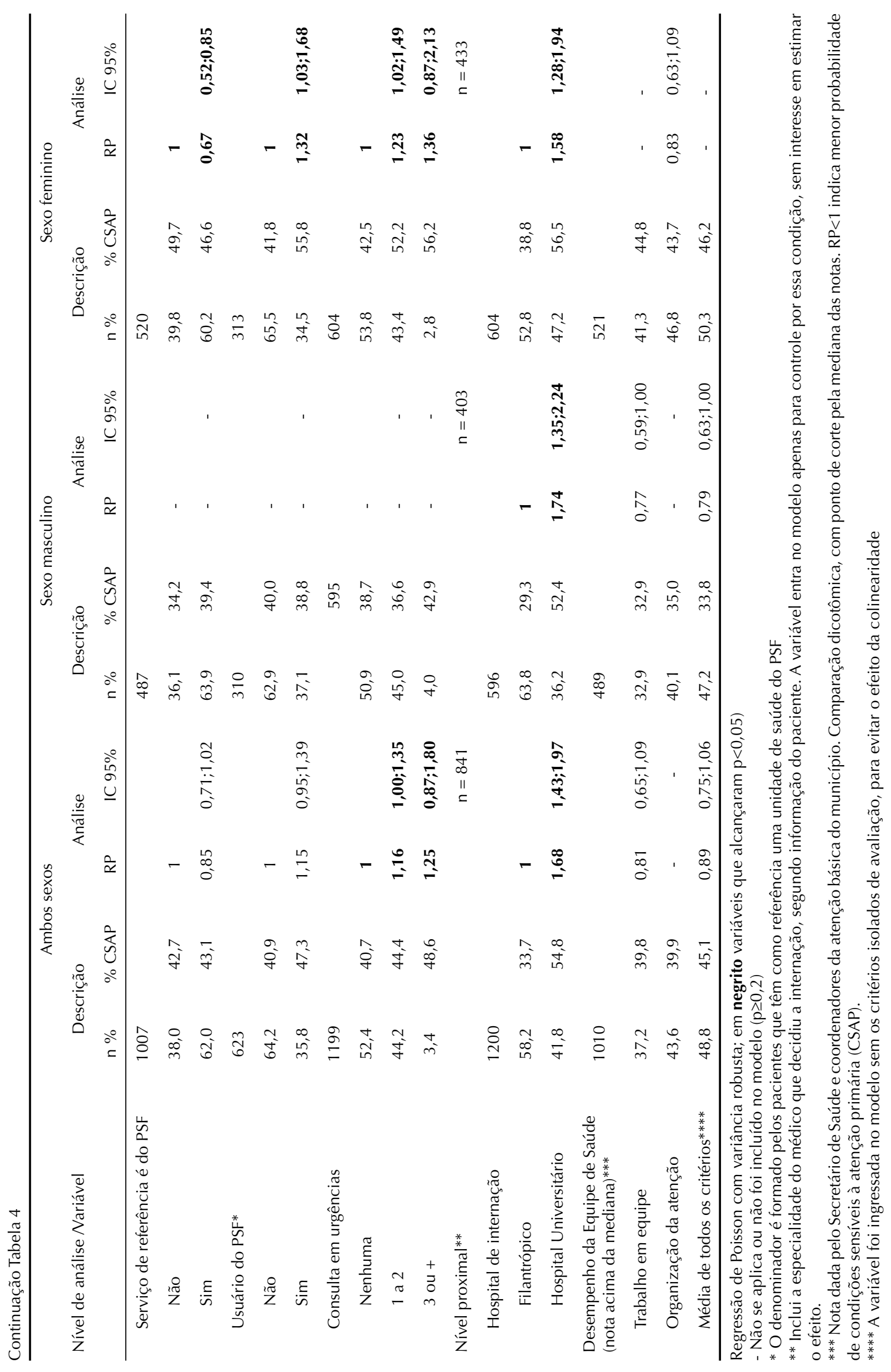


PSF no último mês. No sexo masculino não se observou associação com o modelo de atenção. Entre as mulheres, também associaram-se à prevalência de CSAP: faixa etária, escolaridade, tempo de funcionamento da unidade de saúde, ter como unidade de referência uma Unidade de Saúde da Família, ser usuária do PSF, ter consultado na emergência no último mês e hospital de internação. Para os homens, a faixa etária, ter sofrido outra internação no ano anterior e o hospital de internação.
Ao analisar separadamente o modelo de atenção de referência (Tabela 5), percebem-se algumas singularidades: o sexo só está associado a uma CSAP no PSF e na atenção básica tradicional; nos outros modelos (consulta em especialidade, ambulatório de hospital, sindicato ou empresa, plano de saúde, médico particular, entre outros), a variável de mais forte efeito e significância estatística foi a posse de bens de consumo. Nesses modelos de atenção, não se encontrou associação com o hospital ou

Tabela 5. Análise multivariável de hospitalizações por condições sensíveis à atenção primária por níveis de determinação, segundo o modelo de atenção. Bagé, RS, 2006-2007.*

\begin{tabular}{|c|c|c|c|c|c|c|}
\hline \multirow{3}{*}{ Nível de análise /Variável } & \multicolumn{6}{|c|}{ Modelo de atenção } \\
\hline & \multicolumn{2}{|c|}{$\begin{array}{l}\text { Programa Saúde da } \\
\text { Família }(\mathrm{n}=624)\end{array}$} & \multicolumn{2}{|c|}{$\begin{array}{l}\text { Atenção básica } \\
\text { tradicional }(\mathrm{n}=386)\end{array}$} & \multicolumn{2}{|c|}{ Outro $(n=190)$} \\
\hline & RP & IC 95\% & RP & IC $95 \%$ & $\mathrm{RP}$ & IC $95 \%$ \\
\hline Nível distal & & $\mathrm{n}=586$ & & $\mathrm{n}=356$ & & $\mathrm{n}=184$ \\
\hline Sexo feminino & 1,22 & 1,$02 ; 1,47$ & 1,53 & 1,$19 ; 1,96$ & - & - \\
\hline \multicolumn{7}{|l|}{ Faixa etária (anos) } \\
\hline $5-14$ & 0,74 & 0,$55 ; 0,99$ & 0,32 & 0,$15 ; 0,67$ & 1,20 & 0,$76 ; 1,89$ \\
\hline $15-49$ & 0,53 & 0,$42 ; 0,69$ & 0,54 & 0,$39 ; 0,76$ & 0,53 & 0,$32 ; 0,87$ \\
\hline $50 \mathrm{ou}+$ & 0,65 & 0,$51 ; 0,83$ & 0,60 & 0,$45 ; 0,81$ & 0,72 & 0,$48 ; 1,07$ \\
\hline \multicolumn{7}{|l|}{ Escolaridade (anos) } \\
\hline $5-8$ & 0,91 & 0,$73 ; 1,13$ & 0,73 & 0,$55 ; 0,97$ & - & - \\
\hline $9-11$ & 0,84 & 0,$63 ; 1,12$ & 0,74 & 0,$52 ; 1,04$ & - & - \\
\hline $12 \mathrm{ou}+$ & 0,53 & 0,$15 ; 1,88$ & 0,44 & 0,$16 ; 1,25$ & - & - \\
\hline Classes C, D e E & - & - & - & - & 2,19 & 1,$05 ; 4,59$ \\
\hline Nível intermediário & & $\mathrm{n}=585$ & & $\mathrm{n}=331$ & & $\mathrm{n}=166$ \\
\hline Outra consulta no último mês & - & - & - & - & 1,47 & 1,$07 ; 2,03$ \\
\hline \multicolumn{7}{|l|}{ Hospitalização nos últimos 12 meses } \\
\hline 1 a 2 & - & - & 1,14 & 0,$85 ; 1,52$ & 1,72 & 1,$11 ; 2,65$ \\
\hline $3 \mathrm{ou}+$ & - & - & 1,52 & 1,$14 ; 2,04$ & 2,03 & 1,$35 ; 3,03$ \\
\hline Usuário do PSF** & 1,20 & 1,$00 ; 1,45$ & - & - & - & - \\
\hline \multicolumn{7}{|l|}{ Consulta na emergência } \\
\hline 1 a 2 & 1,29 & 1,$07 ; 1,57$ & - & - & - & - \\
\hline $3 \mathrm{ou}+$ & 1,34 & 0,$82 ; 2,19$ & - & - & - & - \\
\hline \multicolumn{7}{|c|}{ Consulta em plano de saúde ou médico particular } \\
\hline 1 a 2 & - & - & 1,16 & 0,$84 ; 1,61$ & 1,26 & 0,$80 ; 1,96$ \\
\hline $3 \mathrm{ou}+$ & - & - & 1,87 & 0,$72 ; 4,82$ & 2,19 & 0,$96 ; 5,02$ \\
\hline Nível proximal*** & & $\mathrm{n}=571$ & & $\mathrm{n}=331$ & & $\mathrm{n}=166$ \\
\hline Internar no hospital universitário & 1,64 & 1,$37 ; 1,96$ & 1,83 & 1,$37 ; 2,44$ & 1,3 & 0,$88 ; 1,91$ \\
\hline \multicolumn{7}{|c|}{ Desempenho da Equipe de Saúde (Avaliação do gestor) } \\
\hline Trabalho em equipe & 0,84 & 0,$66 ; 1,08$ & - & - & - & - \\
\hline Organização da atenção & - & - & 0,69 & 0,$52 ; 0,92$ & - & - \\
\hline Média de todos os critérios**** & 0,88 & 0,$72 ; 1,07$ & 0,77 & 0,$61 ; 0,98$ & - & - \\
\hline
\end{tabular}

- Não se aplica ou não foi incluído no modelo $(p \geq 0,2)$

* Apresentam-se as variáveis que compõem o modelo em algum dos estratos de análise; omitidas as categorias de referência; em negrito as variáveis que alcançaram $p<0,05$, em itálico as que se mantêm no modelo para ajuste $(p<0,2)$

** Referência: moradores de Área de Saúde da Família que tiveram consulta pelo Programa Saúde da Família no último mês *** O nível 3 (proximal) inclui a especialidade do médico que decidiu a internação, segundo informação do paciente, apenas para controle por essa condição, sem interesse em estimar o efeito. Apresentou $\mathrm{p}=0,02$ para o PSF, $\mathrm{p}=0,12$ para atenção básica $(A B)$ Tradicional, e $p=0,8$ para "Outro", não fazendo parte da análise neste estrato

**** Variável ingressada sem os demais critérios de avaliação, para evitar colinearidade 
médico responsável pela internação. Na mesma Tabela observa-se que a tendência à diminuição das CSAP com o aumento da escolaridade perde significância no grupo coberto pelo PSF. Comparados aos demais pacientes residentes em Área de Saúde da Família, aqueles que consultaram na unidade de saúde no último mês ("usuários") tiveram uma probabilidade $20 \%$ maior de que a causa de internação fosse uma $\operatorname{CSAP}(\mathrm{p}=0,05)$. Consulta na emergência e especialidade do médico da internação só estiveram associados com o desfecho em moradores do PSF. Por outro lado, os critérios de avaliação de desempenho das equipes associaram-se ao desfecho apenas na atenção básica "tradicional".

\section{DISCUSSÃO}

O presente estudo é um inquérito hospitalar, cuja medida de efeito estudada é a proporção de CSAP entre as internações hospitalares, o que não permite inferências sobre o risco de internar por essas causas. Uma população de usuários maior em um determinado modelo de atenção poderia resultar em maior proporção de CSAP nos usuários deste modelo entre os pacientes internados, mesmo com menor taxa de hospitalização.

As associações encontradas apenas podem ser interpretadas como efeito do modelo de atenção quando se referirem a análises dentro de cada modelo (como na proporção de CSAP segundo níveis de escolaridade em cada modelo de atenção), ou quando a análise se referir a toda a população de estudo (como na proporção de CSAP segundo o hospital de internação). Essa pode ser uma explicação para a relação aparentemente paradoxal entre a menor probabilidade de CSAP entre os internados residentes em Área de Saúde da Família e internados usuários do PSF.

Assim, quando o fator de análise exige como denominador a população residente, ou a que consultou por CSAP e não foi internada (como no caso da proporção de CSAP segundo o modelo de atenção), seu efeito poderia ser estimado apenas comparando-se a população hospitalizada com a não-hospitalizada. Para uma incidência de hospitalização por CSAP em torno a $2 \%$ ao ano, uma amostra populacional com tal objetivo teria um orçamento muito alto. A identificação dos denominadores adequados seria possível com a inclusão no Sistema de Informações Hospitalares/SUS de uma variável de registro da UBS de referência do paciente internado e o cadastramento da população residente em cada área de abrangência, a exemplo do que se faz no PSF.

Espera-se na população estudada uma sobre-representação de pessoas com exposições a maiores riscos e em pior condição de saúde que a população geral. A variável "usuário", útil para identificar a parcela da população coberta pelo PSF que não usou o sistema no último mês, talvez incorpore aspectos da situação de saúde do sujeito como a gravidade do problema que gerou a internação, mais que o uso regular do PSF. Tal possibilidade é reforçada por achados do Programa de Expansão e Consolidação da Saúde da Família-UFPel ${ }^{13}$ que mostram os mais pobres, entre os pobres, como usuários da UBS da área de abrangência.

Não se esperam vieses de seleção (além dos devidos a sazonalidade e temporalidade), pois foram entrevistados todos os pacientes internados no período, com pequena proporção de recusas, apesar da maior idade média deste grupo. Não podemos descartar eventuais erros de classificação, sobretudo quando o diagnóstico de internação é influenciado pelo sistema de pagamentos. Entretanto, são notórios os avanços na auditoria do SUS, e os poucos estudos que discutem a confiabilidade diagnóstica da Autorização de Internação Hospitalar concluem pela sua utilidade em estudos epidemiológicos. ${ }^{18}$ Tal viés, se ocorrer, deve ser não-direcional, já que não há porquê esperar diferença em função da UBS de referência do paciente.

Entre outras razões, a hospitalização por CSAP pode acontecer tanto pelo não uso ou uso tardio de recursos oferecidos pela UBS como por manejo clínico inadequado. Entretanto, deve-se considerar que as pessoas buscam soluções a seus problemas de saúde e os profissionais de saúde procuram trabalhar de modo adequado. Como indicadoras da efetividade do sistema de saúde, em quaisquer situações, a interpretação dessas hospitalizações deve ser dirigida às condições de organização do sistema que as geram, não ao usuário ou ao trabalhador de saúde. Por exemplo, o desconhecimento da oferta de serviços e outras barreiras "invisíveis" aos serviços estabelecidos mas percebidas pelo cidadão ou, por outro lado, à má formação dos profissionais de saúde.

As CSAP não avaliam determinantes pessoais da condição clínica do paciente ou a qualidade do ato médico que leva à hospitalização, mas o resultado de políticas e ações executadas frente a tais problemas de saúde. Como feito no presente estudo, variáveis da pessoa podem ser usadas para identificar o cumprimento dos princípios de eqüidade de um modelo de atenção específico, ou para sua inclusão em modelos de análise de variáveis relativas ao sistema de saúde (como proxy das condições de vida das populações assistidas), mas não para inferir responsabilidade ao indivíduo sobre sua necessidade de hospitalização.

A menor probabilidade de CSAP entre as mulheres hospitalizadas que referiram residir em área de abrangência do PSF não ocorreu entre os homens, diferença que pode ser resultado da muito maior utilização dos serviços de atenção primária à saúde pelas mulheres. As associações aparentemente paradoxais observadas nos residentes em área de saúde da família e nos que efetivamente consultaram no PSF no mês anterior à internação podem dever-se, além da falta de um indicador populacional, à possível incorporação da gravidade clínica na variável "usuário". 
É plausível que o serviço consultado antes da hospitalização seja aquele ao qual o indivíduo tem maior vínculo. Entretanto, o desconhecimento da história de uso de serviços impede afirmar que isso ocorra para todos os casos, o que deve ser levado em conta na interpretação dos resultados. Por outro lado, perguntar sobre um período mais amplo possibilitaria maior viés de memória.

Quando o modelo de atenção é a atenção básica do SUS, as principais características da pessoa associadas à internação evitável foram o sexo, idade e escolaridade do paciente. No setor privado e outras formas de organização da atenção, a principal variável associada foi a posse de bens de consumo, mostrando probabilidade maior que o dobro de que a causa de internação seja uma CSAP para os sujeitos de pior condição econômica. Essa característica, que sugere um efeito compensador do SUS sobre a iniqüidade social, é ainda mais evidente no PSF, onde a associação com escolaridade perde significância estatística. O PSF atende mais e recomenda menos a internação que os demais modelos de atenção.

No Brasil, o acompanhamento das hospitalizações evitáveis para avaliação e monitoramento da Atenção Básica foi proposto no Estado do Ceará; estudos promovidos por instituições ligadas às Secretarias Estaduais de Saúde foram realizados na Bahia e Minas Gerais. ${ }^{\text {a,b }}$ O Ministério da Saúde também tem estudado o indicador (de onde resultou a lista de códigos CSAP utilizada no presente estudo), mas não encontramos publicação de estudo brasileiro sobre o tema em revista científica. Este também parece ser o primeiro estudo brasileiro com o indicador integrado usando dados primários não agregados.

A proporção de CSAP encontrada (43\%) é muito maior que a relatada em outros estudos, mesmo entre os espanhóis e australianos (com acesso universal e listas extensas), que variou de $7 \%$ a $13 \%$ do total de internações, ${ }^{2-4,8,10,12}$ embora haja pouca comparabilidade entre as populações e os sistemas de saúde estudados. No exemplo espanhol, com sistema efetivamente universal, foram estudados apenas municípios com uma única "Área Básica de Saúde", o que pode explicar tão baixa proporção desses casos. ${ }^{8}$

Por outro lado a proporção é semelhante à encontrada em Minas Gerais (33\%, contando 20\% de partos, excluídos neste estudo). ${ }^{\text {b }}$ Outros resultados do presente estudo são consistentes com a literatura, que aponta maiores taxas de internação entre a população de pior condição socioeconômica, extremos de idade e variando com a organização e oferta dos serviços, sem muita consistência segundo o sexo. , $, 6,7,16, b^{\text {A }}$ Anão-associação da zona de residência (rural ou urbana) sugere não haver barreiras de acesso à atenção básica ou hospitalar para essa população no município. ${ }^{3}$

Apesar de um efeito incipiente na população estudada, o PSF em Bagé já apresenta sinais de impacto favorável. Não se observa discriminação socioeconômica entre os internados, aproximando-se ao objetivo do princípio de eqüidade. Os resultados observados nas mulheres e na análise por estratos de modelo de atenção sugerem que mesmo com pouco tempo de atividade, o PSF apresenta mais resultados que a atenção básica tradicional. No entanto, as soluções mais imediatas para redução da proporção de CSAP entre as internações do município parecem estar relacionadas à organização da atenção no hospital universitário.

Os critérios de avaliação elaborados com a equipe gestora parecem adequados para a atenção básica tradicional, mas precisam ser melhorados para o PSF. Entretanto, o presente estudo representa apenas a visão desse grupo e não de outros, como os usuários e trabalhadores do sistema.

O indicador ainda é novo e seu conceito está em desenvolvimento, ${ }^{9}$ observando-se uma grande variedade de listas de códigos considerados CSAP e âmbito de aplicação do indicador. O efeito do hospital na determinação da CSAP pode significar a necessidade de adequação teórica da medida. Nesse sentido, a complexidade advinda da multiplicidade de problemas de saúde contabilizados no indicador, com diferente probabilidade de manifestação por sexo e grupo etário e sua relação com os serviços de saúde, indicam um amplo espaço para pesquisa, tanto teórica como de campo, sobre toda a população, ou grupos etários, de sexo e causa, em nível ecológico ou individual de análise de dados.

\section{AGRADECIMENTOS}

À direção e funcionários do Hospital Santa Casa de Caridade de Bagé e do Hospital Universitário da Universidade Regional da Campanha, pela autorização para a pesquisa e pelo apoio dado na identificação dos pacientes internados e de seus registros de alta; ao Secretário Municipal de Saúde de Bagé, Dr. Manif Curi Jorge e gerentes da Atenção Básica da Secretaria Municipal de Saúde de Bagé, pela avaliação das Unidades Básicas de Saúde; aos pareceristas anônimos pelas sugestões.

\footnotetext{
a Mendes EV. A atenção primária à saúde no SUS. Fortaleza: Escola de Saúde Pública do Ceará; 2002.

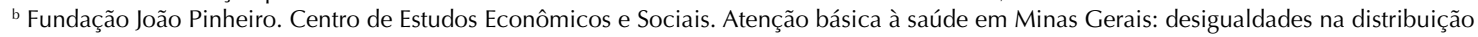
de recursos financeiros e na prestação de serviços básicos após a introdução do piso da atenção básica (PAB) [internet]. Belo Horizonte [citado 2004 nov 21]. Disponível em: http://www.fjp.gov.br/produtos/cees/ Atencao_Basica_em_Minas_Gerais.pdf

c Ministério da Saúde. Departamento de Atenção Básica . Oficina de trabalho para elaboração de uma lista brasileira de hospitalização por condições sensíveis à atenção primária. Belo Horizonte, MG, 9/12/2005. (dados inéditos)
} 


\section{REFERÊNCIAS}

1. Andrade LOM, Barreto ICHC, Goya N, Martins-JR T. Organização da atenção básica e Estratégia Saúde da Família no município de Sobral - Ceará - Brasil: principais avanços e desafios na pesrspectiva de produzir mudanças positivas na saúde. In: Seclen J, Fernandes AS, organizadores. Experiências e desafios da atenção básica e saúde familiar: caso Brasil. Brasília: OPAS; 2004. p.143-79. (Serie tecnica Projeto de Desenvolvimento de Sistemas e Servicos de Saude, 8).

2. Ansari Z, Carson N, Serraglio A, Barbetti T, Cicuttini F. The Victorian Ambulatory Care Sensitive Conditions study: reducing demand on hospital services in Victoria. Aust Health Rev. 2002;25(2):71-7.

3. Ansari Z, Barbetti T, Carson NJ, Auckland MJ, Cicuttini F. The Victorian ambulatory care sensitive conditions study: rural and urban perspectives. Soz Praventivmed. 2003;48(1):33-43. DOI: 10.1007/s000380300004

4. Bermúdez-Tamayo C, Márquez-Calderón S, Rodriguez del Aguila MM, Perea-Milla López E, Ortiz Espinosa J. Caracteristicas organizativas de la atencion primaria y hospitalizacion por los principales ambulatory care sensitive conditions. Aten Primaria. 2004;33(6):305-11. DOI: $10.1157 / 13059762$

5. Billings J, Teicholz N. Uninsured patients ind District of Columbia hospitals. Health Aff (Millwood). 1990;9(4):158-65. DOI: 10.1377/hlthaff.9.4.158

6. Billings J, Zeitel L, Lukomnik J, Carey TS, Blank $\mathrm{AE}$, Newman L. Impact of socioeconomic status on hospital use in New York City. Health Aff (Millwood). 1993;12(1):162-73. DOI: 10.1377/hlthaff.12.1.162

7. Billings J, Anderson GM, Newman LS. Recent findings on preventable hospitalizations. Health Aff (Millwood). 1996;15(3):239-49. DOI: 10.1377/hlthaff.15.3.239

8. Caminal Homar J, Starfield B, Sánchez Ruiz E, Hermosilla Pérez E, Martín Mateo M. La atención primaria de salud y las hospitalizaciones por Ambulatory Care Sensitive Conditions en Cataluña. Rev Clín Esp. 2001;201(9):501-7.

9. Caminal Homar J, Casanova Matutano C. La evaluacion de la atencion primaria y las hospitalizaciones por ambulatory care sensitive conditions. Marco conceptual. Aten Primaria. 2003;31(1):61-5. DOI: 10.1157/13042583

10. Caminal Homar J, Morales Espinoza M, Sanchez Ruiz E, Cubells Larrosa MJ, Bustins Poblet M. Hospitalizaciones prevenibles mediante una atención primaria oportuna y efectiva. Aten Primaria. 2003;31(1):6-14.
11. Caminal J, Mundet X, Ponsà Ja, Sánchez E, Casanova C. Las hospitalizaciones por ambulatory care sensitive conditions: selección del listado de códigos de diagnostico validos para España. Gac Sanit. 2001;15(2):128-41.

12. Caminal J, Sánchez E, Morales M, Peiro R, Marquez S. Avances en Espana en la investigación con el indicador "Hospitalizacion por Enfermedades Sensibles a Cuidados de atención primaria". Rev Esp Salud Publica. 2002;76(3):189-98. DOI: 10.1590/ S1135-57272002000300003

13. Facchini LA, Piccini RX, Tomasi E, Thumé E, Silveira DS, Siqueira FV, et al. Desempenho do PSF no Sul e no Nordeste do Brasil: avaliação institucional e epidemiológica da Atenção Básica à Saúde. Cienc Saude Coletiva. 2006;11(3):669-81. DOI: 10.1590/ S1413-81232006000300015

14. Machado ENM Fortes FBCTP, Somarriba M. Efeitos da introdução do PAB sobre a distribuição de recursos e a prestação de serviços: o caso de Minas Gerais. Cienc Saude Coletiva. 2004;9(1):99-111. DOI: 10.1590/ S1413-81232004000100010

15. Macinko J, Almeida C, Oliveira dos SE, de Sá PK. Organization and delivery of primary health care services in Petropolis, Brazil. Int J Health Plann Manage. 2004;19(4):303-17. DOI: 10.1002/hpm.766

16. Márquez-Calderón S, Rodríguez del Aguila MM, Perea-Milla E, Ortiz J, Bermúdez-Tamayo C. Factores asociados a la hospitalizacion por procesos sensibles a cuidados ambulatorios en los municipios. Gac Sanit. 2003;17(5):360-7. DOI: 10.1157/13053648

17. Marrugat J, Vila J, Pavesi M, Sanz F. Estimación del tamaño de la muestra en la investigación clínica y epidemiológica. Med Clin (Barc). 1998;111(7):267-76.

18. Mathias TAF, Soboll MLMS. Confiabilidade de diagnósticos nos formulários de autorização de internação hospitalar. Rev Saude Publica. 1998;32(6):526-32. DOI: 10.1590/S003489101998000600005

19. Pacto de Indicadores da Atenção Básica: instrumento de negociação qualificador do processo de gestão do SUS. Rev Bras Saude Matern Infant. 2003;3(2):221-4.

20. Weissman JS, Gatsonis C, Epstein AM. Rates of avoidable hospitalization by insurance status in Massachusetts and Maryland. JAMA. 1992;268(17):2388-94. DOI: 10.1001/ jama.268.17.2388

Artigo apresentado ao Programa de Pós-Graduação em Epidemiologia da Universidade Federal de Pelotas para obtenção do grau de Doutor.

Financiado pelo Conselho Nacional de Desenvolvimento Científico e Tecnológico e Secretaria de Assistência à Saúde por meio do Edital MCT-CNPq / MS-DAB/SAS - No 49/2005.

FB Nedel foi apoiado pela Coordenação de Aperfeiçoamento de Pessoal de Nível Superior (Capes; bolsa de doutorado). 